Relations industrielles

Industrial Relations

\title{
SMITH, May, "An Introduction to Industrial Psychology", Cassell and Company Ltd., London 1952, 295 pp.
}

\section{Fabienne Tousignant}

Volume 9, numéro 1, décembre 1953

URI : https://id.erudit.org/iderudit/1022922ar

DOI : https://doi.org/10.7202/1022922ar

Aller au sommaire du numéro

Éditeur(s)

Département des relations industrielles de l’Université Laval

ISSN

0034-379X (imprimé)

1703-8138 (numérique)

Découvrir la revue

Citer ce compte rendu

Tousignant, F. (1953). Compte rendu de [SMITH, May, "An Introduction to Industrial Psychology", Cassell and Company Ltd., London 1952, 295 pp.] Relations industrielles / Industrial Relations, 9(1), 87-88.

https://doi.org/10.7202/1022922ar

Tous droits réservés (C Département des relations industrielles de l’Université Laval, 1953
Ce document est protégé par la loi sur le droit d'auteur. L’utilisation des services d'Érudit (y compris la reproduction) est assujettie à sa politique d'utilisation que vous pouvez consulter en ligne.

https://apropos.erudit.org/fr/usagers/politique-dutilisation/ 
SMITH, MAY, “An Introduction to Industrial Psychology", Cassell and Company Ltd., London 1952, 295 pp.

Ce livre fait suite à la lère édition de ce volume parue au cours de 194142; il a pour but non de régler les problèmes soulevés dans l'industrie mais de donner quelque lumière sur la nature de ces problèmes à ceux qui sont intéressés de près ou de loin au travail des autres ou qui sont en relation immédiate avec les travaileurs. L'auteur en contact elle-même avec diverses organisations industrielles et autres a été à même de connaître les individus de ces divers groupes.

Depuis longtemps avec les développements remarquables de l'industrie, certains penseurs avaient réalisé l'importance croissante des problèmes de la vie industrielle. Ce milieu de l'industrie devenait un champ d'application de la psychologie qui devait revêtir un caractère particulier et spécifique pour s'arrêter à l'homme-travailleur. La psychologie industrielle est définie par l'auteur comme «l'étude de la conduite de ceux qui échangent le travail de leur esprit et de leurs mains pour obtenir des moyens de subsistance de vie $\gg$; ceci inclut une grande partie de la population.

Au début de son étude, Miss Smith fait une revision historique du travail de quelques pionniers dans ce domaine, au point de vue technique, médical et enfin humain. Tout un chapitre est consacré à l'étude de la fatigue; comme il s'agit de fatigue industrielle, il est important de saisir comment elle se manifeste et dans quelles circonstances. On peut trouver d'intéressantes expériences tentées dans différents milieux de travail et sous diverses conditions physiques et psychologiques.

Comme le travailleur passe la majeure partie de sa vie à l'usine, au bureau, il est normal que l'auteur se soit arrêtée à son entourage: entourage matériel d'abord comportant: éclairage, température, bruit, heures de travail et ait appuyé ses observations d'opinions d'ouvriers et d'employés cléricaux. L'atmosphère psychologique créée par le groupe, la monotonie, lautorité, joue un rôle très important sur la conduite et la pensée de l'individu. Toutefois, il est à noter que les réactions ne se font pas sentir de la même façon chez tous et chacun; cela oblige donc ceux qui ont des employés à charge à tenir compte des différences, des goûts, des aptitudes et des tempéraments de chacun. Pour donner la plus grande satisfaction au travailleur à son travail comme l'explique si justement Miss Smith, et pour qu'une plus grande harmonie au lieu du travail puisse exister, il s'agit d'éviter l'apparition de griefs nuisant aux bonnes relations. Le choix judicieux et l'orientation adéquate de chaque individu suivis d'une entraînement élaboré et intelligent peuvent être la prévention de maux et la solution à bien des conflits. Elle insiste également sur la possibilité de promotion.

L'étude des temps et des mouvements faite de façon apropriée et juste peut être applicable et adaptée aux travailleurs. Il s'agit de toujours et en tout temps tenir compte des tempéraments, surtout les nerveux, et d'apporter beaucoup de compréhension.

Le chapitre portant sur le travail, les motifs de travailler est d'un intérêt particulier et démontre jusqu'à quel point, il ne faut pas oublier le côté humain du travailleur qui a ses besoins, ses goûts et ses aspirations légitimes. Le premier motif est l'argent à recevoir qui satisfait ses nécessités et le rend indépendant dans la société et lui permet de vivre une vie normale d'homme; l'ouvrier a également au milieu du travail, auprès de ses compagnons, égaux, et supérieurs une réponse à ses sentiments d'homme et une chance de pouvoir s'exprimer personnellement et de satisfaire son ambition; il peut exercer, de plus, son habileté et développer ses talents et occuper son corps et son esprit à un travail utile plutôt que de perdre son temps. Toujours, l'ouvrier recherche la sécurité, des conditions de travail confortables, de bons chefs, des chances d'avancement, de hauts salaires, des heures courtes et du travail qui ne requiert pas trop d'effort de pensée. Dans ces conditions l'employé est satisfait et c'est à l'autorité de voir à ce qu'il puisse trouver à l'industrie une réponse satisfaisante.

Les derniers chapitres ont trait aux accidents, griefs, maladies, départs, cas d'exceptions, méthodes à appliquer; toujours l'auteur s'attache à découvrir d'une façon ou d'une autre les causes de telle situation, les moyens de l'améliorer en tenant compte de la person- 
nalité de chaque individu et surtout du côté humain de sa personne. C'est d'ailleurs en ne perdant pas ce point de vue que des enquêtes pourront être faites de façon utile et apporter des résultats satisfaisants.

Ce livre offre une grande valeur à tous ceux qui s'occupent de façon médiate ou immédiate de psychologie industrielle $e$ : s'attachent particulièrement à l'homme dans la personne du travailleur.

Fabienne Tousignant

\section{PUBLICATIONS RÉCENTES}

\section{Direction du personnel}

Building a Functional Personnel Budget, by Dale Yoder from The Management Review published by American Management Association, 330 West 42nd Street, N.Y. 36, September 1953, pp. 504-513.

Communication in Industry: A cure of conflict by Paul Pigors from Industrial and Labor Relations Review - New York State School of Industrial and Labor Relations, Cornell University, Ithaca, N.Y. - July 1953, p. 496-509.

L'employé bien renseigné est un meilleur employé par J.A. Fuller, causerie prononcée à la Cinquième Conférence annuelle de la Canadian Industrial Trainers' Association, 1951, 10 pp.

The Well-Informed Employee is a Better Employee by J.A. Fuller, address given to the Annual Convention of the Canadian Industrial Trainers' Associa(ion, $1951,10 \mathrm{pp}$.

Employee Record Cards by Richard Lane, article from the Factory Manager mublished by Production Publications (London) Limited, 117 Kingsway, London, W.C. 2, Septembre 1953, pp. 52.

Guidance for Management, article in Industrial Bulletin published by New York State Department of Labor, 80 Centre Stree', New York 13, N.Y., November 1953, pp. 10-I5.
La méthode des tests, ses avantages et ses limites, par Charles Mertens de Wilmars, article de «Industrie », revue de la Fédération des Industries Belges, 33, rue Ducale, Bruxelles, octobre 1953, pp. 644-648.

Management Communication and the Gravepine by Keith Davis from Harvard Business Review, Soldiers Field, Boston 63, Massachusetts, September-October 1953, pp. 43 à 50.

What Employees want to knowabout Job Evaluation? Article from "Plant Administration", Maclean-Hunter publishing Co. Ltd., 481 University Avenue, Toron'o, November 1953, pp. 72-74.

L'organisation scientifique dans les entreprises de transport automobile par Gaston Erard, article extrait de «Travail et méthodes », publié par Editions Science et Industrie, Service Documentation, 6, ave Pierre ler de Serbie, Paris (16è), pp. 21-33.

The Third Phase of Personnel Adminisiration by Lawrence A. Appley, article from Management News published by the American Management Association, 330 West 42 St., New York 36, September 1953.

\section{Généralités}

A New Conception of Leadership by Norman Clark, article from the Factory Manager, published by Production $\mathrm{Pu}$ blications (London) Limited, 117 Kingsway, London W.C. 2, September 1953, pp. 57.

L'organisation professionnelle, J.-P. Delarge et J. Lannove, ariicle du Bulletin Social des Industriels, 71, avenue de Cortenberg, Bruxelles, pp. 417-321, novembre 1953.

Recherches sur l'opinion publique publié dans le vol. 5, no 3, 1953, du Bulletin international des Sciences Sociales, Unesco, 19, avenue Kléber, Paris 16è, $210 \mathrm{pp}$.

\section{Industrie}

An Industrial Problem, Australia's Longshoremen by Mark Perlman, Reprint Series Number 16, published by New York State School of Industrial and Labor Relations at Cornell University, Ithaca, N.Y., 11 pp., 1953.

Emploi et salaires dans l'industrie de l'imprimerie 1943-1952, publié par le 